\title{
Effect of a Clathrate-Forming Counterion on Micellar Solubilization
}

\author{
Emily Ogle, Clifford E. Larrabee, Jr. \\ University of Cincinnati \\ Cincinnati, Ohio, USA \\ ogleea@mail.uc.edu; cliff.larrabee@uc.edu
}

\begin{abstract}
Clathrate hydrates are supramolecular solids in which small, hydrophobic species are trapped inside a network of hydrogen-bonded water cages. While the best known examples are the natural gas hydrates, similar crystalline structures can also be formed from salts of tetra- $n$-butylammonium hydroxide (TBAOH). When TBAOH is reacted with medium to long chain carboxylic acids it produces surfactant compounds capable of forming either clathrate hydrates or micelle solutions in aqueous solution. However, little is known about how this dual nature affects the functional properties of the surfactants. Here we show a direct comparison of the solubilization of the solvatochromic dye, Nile Red (NR) by sodium 10-undecenoate (NaU) and tetrabutylammonium 10-undecenoate (TBAU). We found from the fluorescence emission spectra that the environment around NR was more polar for TBAU micellar solutions than for NaU. Molecular dynamics simulations indicated that for both systems, the NR had partial exposure to water at the surface of the micelle. The primary difference was a significant structuring of the counterions and water molecules as part of the micelle/NR complex for TBAU. Our results demonstrate an active role of role of the clathrate-forming counterion in the formation of the micelle and the solubilization of the substrate. We anticipate that the more polar environment near the surface of the micelle induced by the TBA/ $\mathrm{H}_{2} \mathrm{O}$ structuring will enhance the solubilization power of the micelle for more polar substrates. Furthermore, we expect the more structured shell around the micelle core to enhance the isolation of the substrate from the environment, a valuable feature for applications such as targeted drug delivery.
\end{abstract}

Keywords: micelle, solubilization, clathrate, fluorescence spectroscopy, molecular dynamics, model system, targeted drug delivery

\section{Introduction}

Micelles form from amphiphilic surfactant compounds in aqueous solution by aggregating with their hydrophobic tails in a roughly spherical core surrounded by a shell composed of their hydrophilic head groups. For ionic compounds, the hydrophilic shell is surrounded by an inner, ionic Stern layer, and a more diffuse, ionic Gouy-Chapman layer. Lipophilic substrates are solubilized in the hydrophobic "pseudo phase" of the micelle cores. [1]

Clathrate hydrate formation is governed by the same hydrophobic interactions that govern micelle assembly, but since the solute particles tend to be small, the water cavities surrounding them are also small. [2] The water cavities also exhibit structured, regular polyhedra composed of fully hydrogen-bonded water pentagrams and hexagrams connected in a supramolecular network. The best known examples are the natural gas hydrates that have important energy and environmental applications. [3] Closely related clathrate-hydrate crystal structures can also be formed from salts of tetra- $n$ butylammonium (TBA) hydroxide, where each butyl group supports the structure of one water cage. [4], [5], [6]

At this time, the number of compounds known to promote both micelle and clathrate hydrate formation is small and the number of studies of their dual nature is limited. [7] Micelles of TBA carboxylates exhibit unusually high solubility and small aggregation numbers [8], and relatively high solubilization power [7]. Micelles of TBA perfluorooctanoate have exceptional thermodynamic properties. [9]

The overall goal of this research is to develop a better understanding of the role of a clathrate-forming counterion on the structure and function of aqueous, ionic micelles. The specific objective of the work is to investigate the molecular structure of the micelle/substrate complex formed from tetra-n-butylammonium 10-undecenoate and the lipophilic dye, Nile Red, NR (The chemical structure of Nile Red is shown in figure 1. 


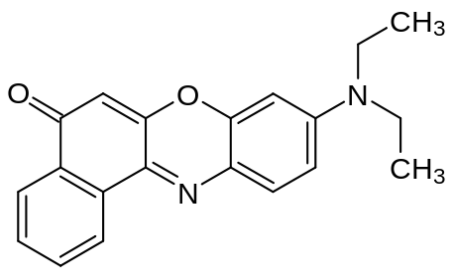

Fig. 1: Nile Red (NR).

The 10-undecenoate anion was selected because its micelles are well characterized [10], polymerizable [11], and its oligomers have shown potential as a model for targeted drug delivery systems [12]. The solubilization power, and $\mathrm{pH}-$ mediated release of lipophilic substrates from TBAU micelles are also relevant features for a drug delivery system model [7], [13] Finally, Nile Red is a lipophilic substrate with solvatochromic properties suitable for describing the polar environment of the micelle. [14], [15]

\section{Materials and Methods}

\subsection{Materials}

All solutions were made up with reagent grade, ASTM Type II water (Ricca Chemical Company). Stock solutions of sodium and tetrabutylammonium 10-undecenoate were prepared by neutralizing 10-undecenoic acid (Acros, 99\%) with sodium hydroxide (Fisher NF/FCC) or tetrabutylammonium hydroxide (Acros, $40 \%$ in water) after standardizing the base with potassium hydrogen phthalate (Fisher, ACS). Nile Red (Sigma Aldrich) was technical grade. Methanol (PharmcoAaper), ethanol (Acros), proponal (Fisher), butanol (Fisher), pentanol (Matheson, Coleman \& Bell), and octanol (Acros) were all reagent grade or better. All substances were used as received, without further purification.

\subsection{Fluorescence Spectroscopy}

Fluorescence spectra were taken in a temperature-controlled lab $(295.0 \pm 0.8 \mathrm{~K})$ with a Vernier Spectrovis Plus spectrophotometer. All samples were filtered (GE Whatman PVDF $0.45 \mu \mathrm{m}$ ) into glass cuvettes and measured at room temperature using an excitation frequency centered at $500 \mathrm{~nm}$. The manufacturer's specifications for accuracy and precision are $3.0 \mathrm{~nm}$ and $1.0 \mathrm{~nm}$ respectively.

\subsection{Molecular Dynamics}

All simulations employed Gromos 54a7 force fields [16], calculated with the Automated Topology Builder at the University of Queensland, Brisbane, Australia [17], [18], [19], and the simple point charge (SPC) water model [20]. Micelle/substrate complexes were preformed with Packmol [21], [22], The aggregation numbers for the preformed micelles were 46 for $\mathrm{NaU}$ [10] and 26 for TBAU, extrapolated from [8]. Both systems were placed in dodecahedral boxes with $2.0 \mathrm{~nm}$ from the micelle to the faces of the box, solvated, provided with sodium ions (sodium 10-undecenoate, NaU, micelles), and equilibrated before the final production runs of $50 \mathrm{~ns}$ at $300 \mathrm{~K}$ (Gromacs 5.1.4 [23], [24], [25], [26]). Radial distribution functions were determined for the final $20 \mathrm{~ns}$ of each simulation with the Gromacs program, gmx rdf [27] and the snapshots were obtained with the molecular visualization program, VMD [28].

\section{Results}

\subsection{Fluorescence Spectroscopy}

Fluorescence spectra of NR in various normal alcohols were taken to correlate the wavelength of maximum peak intensity, $\lambda \max$, with relative polarity of the solvent [29]. The results, plotted in Figure 2 show a very good correlation, R2 $=0.98$. In addition, the measured value of $\lambda \max$ for methanol, $638.7 \mathrm{~nm}$ is in good agreement with the value of $637.9 \mathrm{~nm}$ reported by Stuart, et al. [14]. 


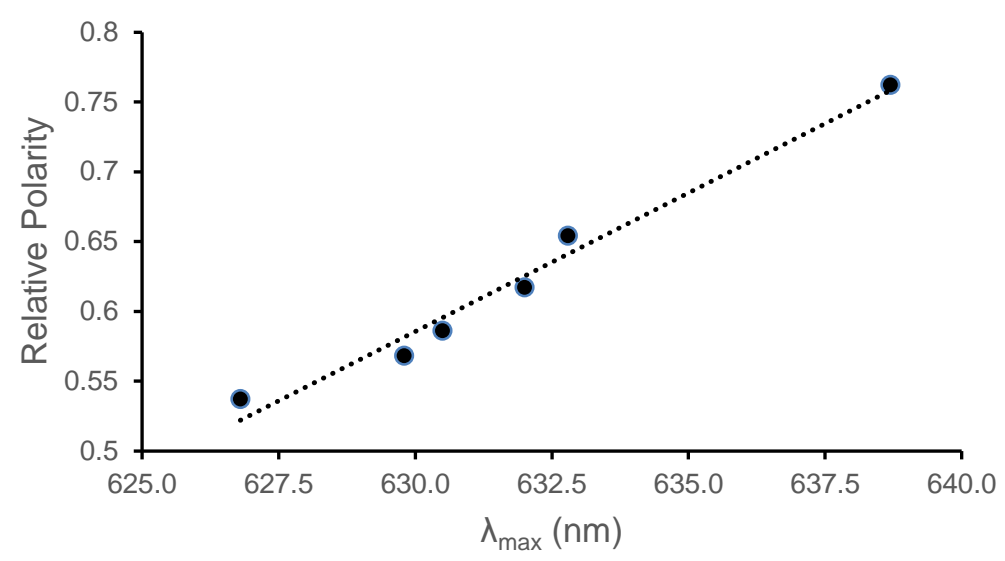

Fig. 2: Relative polarity of the solvent [29] vs. Nile Red (NR) wavelength of maximum emission. The points, from left to right, correspond to 1-octanol, 1-pentanol, 1-butanol, 1-propanol, ethanol, and methanol.

Fluorescence spectra for NR in NaU and TBAU micelle solutions are shown in Figure 3. For each micelle system, the values of $\lambda_{\max }$ did not depend significantly on concentration. The value of $\lambda_{\max }$ for NR in NaU micelles, $630.4 \mathrm{~nm}$, is very close to that of butanol, $630.5 \mathrm{~nm}$, indicating a relative polarity of 0.59 compared to a value of 1.00 for water. The value of $\lambda_{\max }$ for NR in TBAU micelles, $638.5 \mathrm{~nm}$, is very close to that of methanol, $638.7 \mathrm{~nm}$, indicating a relative polarity of 0.76 .
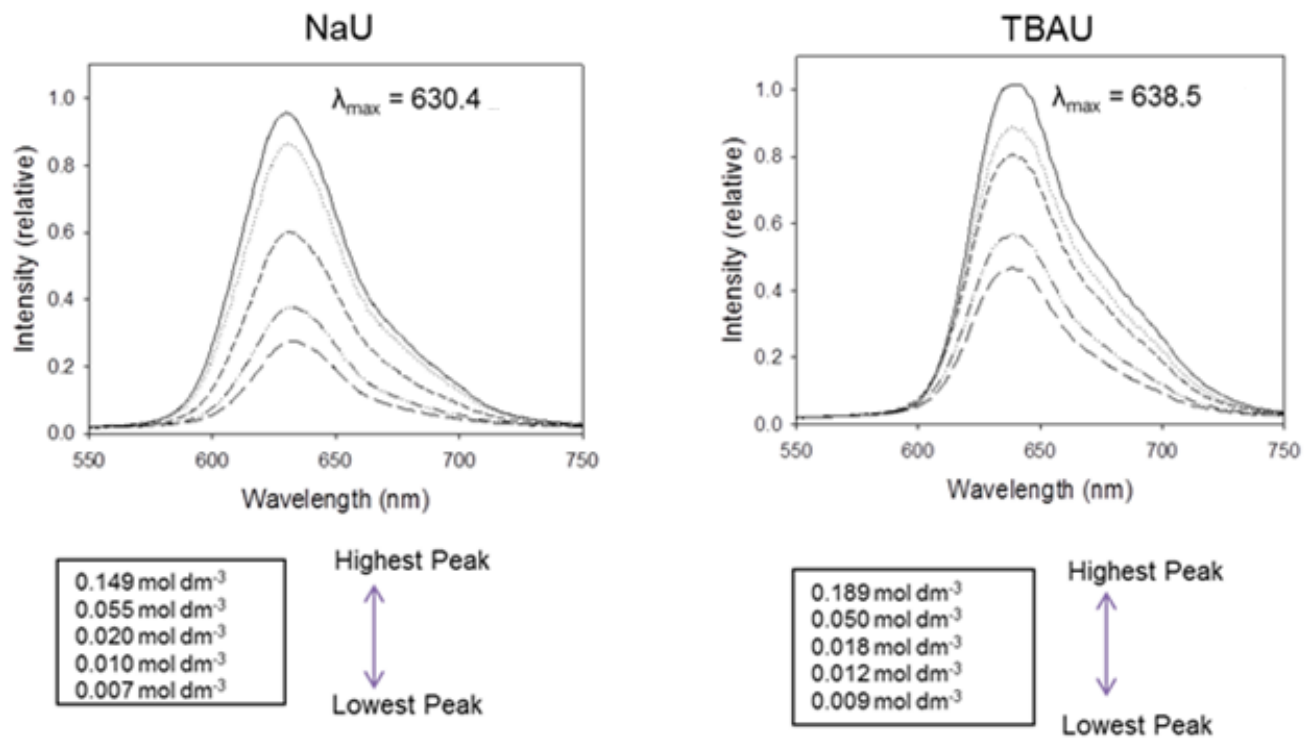

Fig. 3: Fluorescence spectra of Nile Red (NR) in sodium 10-undecenoate (NaU) micelle solution (left) and in tetrabutylammonium 10undecenoate (TBAU) micelle solution (right). For each micelle system, the fluorescence intensity increased with increasing surfactant concentration while the wavelength of maximum intensity remained constant.

\subsection{Molecular Dynamics}

The snapshots of the molecular dynamics simulations in Figure 4 illustrate the qualitative differences between the two micelle/substrate complexes. Both systems show compact, roughly spherical micelles with partial exposure of the substrate at the surface. For the $\mathrm{NaU}$ micelle, the sodium counterions can be observed close to the micelle surface, Stern layer, as well as distributed in the more diffuse Gouy-Chapman layer. In contrast, most of the TBA ions in the TBAU micelle appear to be an integral part of the micelle surface structure. This is attributed to the hydrophobicity of the TBA counterion. 

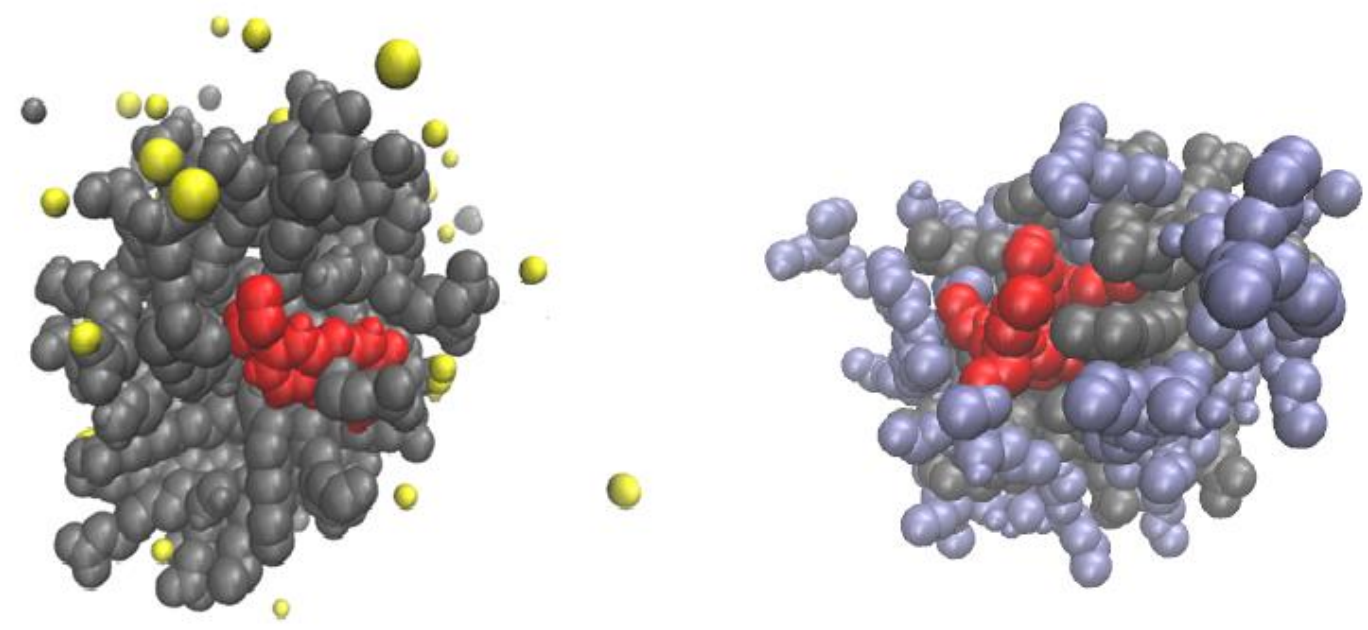

Fig. 4: Snapshots of simulations of micellar solubilization of Nile Red (red). The image on the left shows the sodium (yellow) 10undecenoate (grey) micelle and the image on the right shows the tetrabutylammonium (blue) 10-undecenoate (grey) micelle. For clarity, water molecules are not shown in these images.

The radial distribution functions for the two counterions relative to the NR substrate are shown in Figure 5, where g(r) is the probability of finding any atom of the counterion at a distance, $r$, from any atom of the Nile Red. The diffuse counterion distribution for sodium ions observed in Figure 4 are indicated quantitatively in this figure with weak peaks at about $0.5 \mathrm{~nm}$ (Stern layer) and about $1 \mathrm{~nm}$ (Gouy-Chapman layer). In contrast, the distribution for TBA ions shows two prominent peaks: an inner peak at $0.50 \mathrm{~nm}$ and a secondary peak at $0.84 \mathrm{~nm}$. The well-defined peaks in the radial distribution function for TBA indicate a high degree of structuring for the counterions in this system.

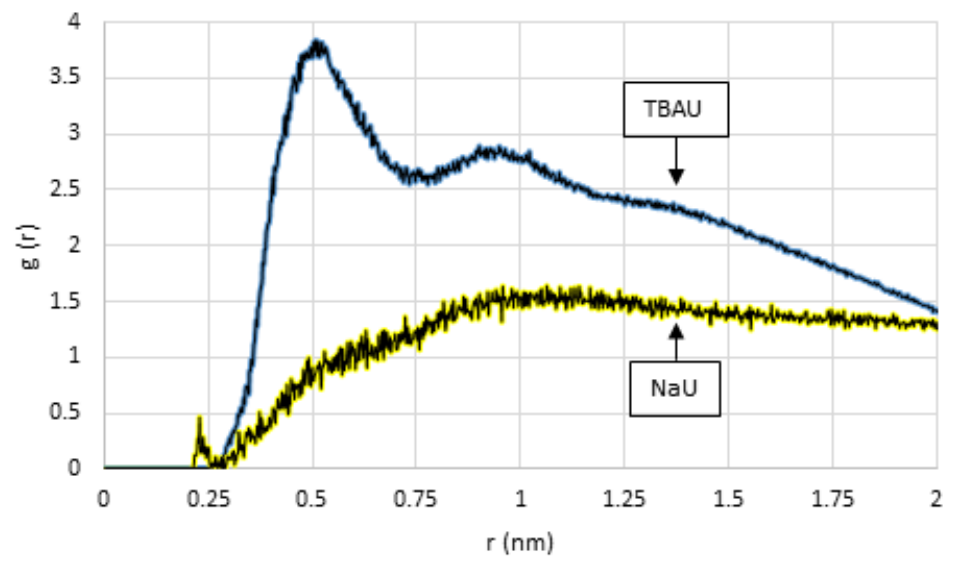

Fig. 5: Radial distribution functions of the counterions about the Nile Red (NR) substrate. The sodium ion distribution is represented by the yellow line and the tetrabutylammonium ion distribution is represented by the blue line.

The radial distribution functions for water relative to the NR substrate in the two micelle systems are shown in Figure 6, where $g(r)$ is the probability of finding any atom of water at a distance, r, from any atom of the Nile Red. Again, there is very little structuring apparent for the NaU micelles, while it is pronounced in the TBAU micelles. The peak for water at $0.45 \mathrm{~nm}$ is just inside the TBA peak at $5.0 \mathrm{~nm}$. The valley at $0.55 \mathrm{~nm}$ is just outside. 


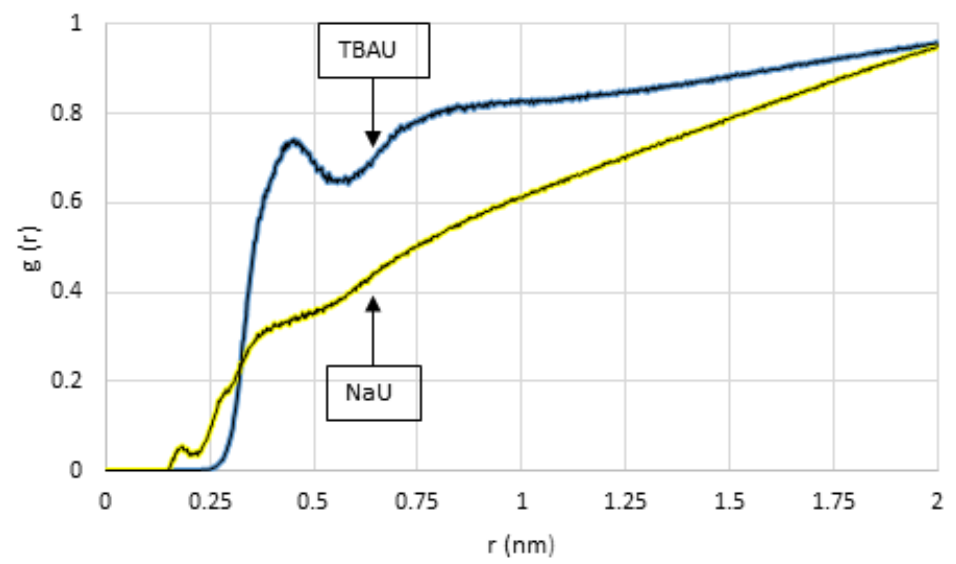

Fig. 6: Radial distribution functions of water about the Nile Red substrate. The NaU water distribution is represented by the yellow line and the TBAU water distribution is represented by the blue line.

We believe that the correspondence of the counterion and water radial distribution functions for the TBAU micelles and the lack of similar structuring of either the counterion or water in the NaU micelles is compelling evidence that the TBA counterions cause the structuring of water at the surface of the TBAU micelles. This is consistent with the known TBA/water structuring in clathrate hydrate formation. [4], [5], [6] We believe this is not an artefact of the simulation model since it agrees with our observation of increased polarity of the environment near NR in TBAU micelles from the fluorescence spectroscopy experiments.

\section{Conclusion}

The picture that emerges for the sodium 10-undecenoate, $\mathrm{NaU}$, micelle conforms to the standard model of a roughly spherical micelle surrounded by diffuse, but identifiable Stern and Gouy-Chapman layers. The picture of the tetrabutylammonium, TBAU, micelle is fundamentally different: the counterions are an integral part of the micelle, forming an outer shell and entrapping water molecules as part of the shell.

The packing of the TBA ions into the shell of the micelle may explain, in part, why the aggregation numbers of TBA micelles is unusually low. The hydrocarbon component of the micelle now includes a significant contribution from the counterion. The trapping of water within the micelle's shell may be involved in the unusual thermodynamics of TBA micelles. The restructuring of water on TBA micelle formation almost certainly will affect both the enthalpy and entropy of micellization.

1. Two types of applications of micelles incorporating structured counterion/water shell can be foreseen:

The polar nature of the shell should change the solubilization characteristics of the micelle, possibly increasing the solubilization power of the micelle for substrates with hydrophilic moieties. The shell should isolate any substrate from interaction with the environment. This would be desirable in the design of drug delivery systems.

2. The shell should isolate any substrate from interaction with the environment.

Finally, it should be noted that the counterion/water shell, like the micelle itself is dynamic. At this temperature, the tetrabutylammonium 10-undecenoate, TBAU, micelles are very micelle-like. There is no evidence of a crystalline, clathrate-like structure for the micelle or the shell.

\section{Acknowledgements}

Emily Ogle was supported in part by the University of Cincinnati, Women in Science and Engineering (WISE) program. The molecular force fields were calculated at the University of Queensland, Brisbane, Australia. 


\section{References}

[1] C. Tanford, The Hydrophobic Effect: Formation of Micelles and Biological Membranes 2d Ed. J. Wiley., 1980.

[2] D. Chandler, "Interfaces and the driving force of hydrophobic assembly," Nature, vol. 437, no. 7059, pp. 640-647, 2005.

[3] E. D. Sloan Jr and C. Koh, Clathrate hydrates of natural gases. CRC press, 2007.

[4] Y. A. Dyadin, L. Gaponenko, L. Aladko, and S. Bogatyryova, "Clathrate hydrates of tetrabutylammonium carboxylates and dicarboxylates," in Clathrate Compounds, Molecular Inclusion Phenomena, and Cyclodextrins: Springer, pp. 259-266, 1984.

[5] Y. A. Dyadin and L. S. Aladko, "Clathrate Hydrates of Long-chain Tetrabutylammonium Carboxylates," Mendeleev Communications, vol. 5, no. 6, pp. 239-240, 1995.

[6] T. Rodionova, V. Komarov, J. Lipkowski, and N. Kuratieva, "The structure of the ionic clathrate hydrate of tetrabutylammonium valerate (C 4 H 9) 4 NC 4 H 9 CO 2. 39.8 H 2 O," New Journal of Chemistry, vol. 34, no. 3, pp. 432-438, 2010.

[7] C. E. Larrabee Jr, M. R. Warmin, and M. J. Howard, "Clathrate Hydrate Formation and Micellization of Tetrabutylammonium 10-Undecenoate," (in English), International Journal of Theoretical and Applied Nanotechnology, vol. 2, pp. 40-45, 20142014.

[8] R. Zana, "Partial phase behavior and micellar properties of tetrabutylammonium salts of fatty acids: Unusual solubility in water and formation of unexpectedly small micelles," Langmuir, vol. 20, no. 14, pp. 5666-5668, 2004.

[9] C. Wang, P. Yan, H. Xing, C. Jin, and J.-X. Xiao, "Thermodynamics of Aggregation of Ammonium/Tetraalkylammonium Perfluorooctanoates: Effect of Counterions $\dagger$," Journal of Chemical \& Engineering Data, vol. 55, no. 5, pp. 1994-1999, 2010.

[10] J. M. Denton, D. C. Duecker, and E. D. Sprague, "Size and solution behavior of sodium 10-undecenoate oligomers," The Journal of Physical Chemistry, vol. 97, no. 3, pp. 756-762, 1993.

[11] C. E. Larrabee Jr and E. D. Sprague, "Radiation-induced polymerization of sodium 10-undecenoate in aqueous micelle solutions," Journal of Polymer Science: Polymer Letters Edition, vol. 17, no. 12, pp. 749-751, 1979.

[12] C. E. Larrabee Jr, M. R. Warmin, and C. N. Iles, "Effect of Oligomerization of Sodium 10-Undecenoate on the Solubilization of a Hydrophobic Substrate," in International Conference on Nanotechnology: Fundamentals and Applications, Prague, Czech Republic, 2014, vol. 5, pp. 104-1-4: Avestia, 2014.

[13] M. Robison, M. R. Warmin, and C. E. Larrabee Jr, "pH-Mediated Release in a Model Drug Delivery System," in Proceedings of the 2nd World Conference on Nanotechnologies (NewTech'16), Budapest, Hungary, 2016, pp. 1091-6: Avestia, 2016.

[14] M. C. Stuart, J. C. van de Pas, and J. B. Engberts, "The use of Nile Red to monitor the aggregation behavior in ternary surfactant-water-organic solvent systems," Journal of physical organic chemistry, vol. 18, no. 9, pp. 929934, 2005.

[15] I. N. Kurniasih, H. Liang, P. C. Mohr, G. Khot, J. r. P. Rabe, and A. Mohr, "Nile red dye in aqueous surfactant and micellar solution," Langmuir, vol. 31, no. 9, pp. 2639-2648, 2015.

[16] N. Schmid et al., "Definition and testing of the GROMOS force-field versions 54A7 and 54B7," European biophysics journal, vol. 40, no. 7, pp. 843-856, 2011.

[17] A. K. Malde et al., "An automated force field topology builder (ATB) and repository: version 1.0," Journal of chemical theory and computation, vol. 7, no. 12, pp. 4026-4037, 2011.

[18] S. Canzar et al., "Charge group partitioning in biomolecular simulation," Journal of Computational Biology, vol. 20, no. 3, pp. 188-198, 2013.

[19] K. B. Koziara, M. Stroet, A. K. Malde, and A. E. Mark, "Testing and validation of the Automated Topology Builder (ATB) version 2.0: prediction of hydration free enthalpies," Journal of computer-aided molecular design, vol. 28, no. 3, pp. 221-233, 2014.

[20] H. J. Berendsen, J. P. Postma, W. F. van Gunsteren, and J. Hermans, "Interaction models for water in relation to protein hydration," in Intermolecular forces: Springer, pp. 331-342, 1981.

[21] J. M. Martínez and L. Martínez, "Packing optimization for automated generation of complex system's initial configurations for molecular dynamics and docking," Journal of computational chemistry, vol. 24, no. 7, pp. 819$825,2003$. 
[22] L. Martínez, R. Andrade, E. G. Birgin, and J. M. Martínez, "PACKMOL: a package for building initial configurations for molecular dynamics simulations," Journal of computational chemistry, vol. 30, no. 13, pp. 2157-2164, 2009.

[23] W. F. van Gunsteren and H. J. Berendsen, "Computer simulation of molecular dynamics: Methodology, applications, and perspectives in chemistry," Angewandte Chemie International Edition in English, vol. 29, no. 9, pp. 992-1023, 1990.

[24] H. J. Berendsen, D. van der Spoel, and R. van Drunen, "GROMACS: a message-passing parallel molecular dynamics implementation," Computer Physics Communications, vol. 91, no. 1, pp. 43-56, 1995.

[25] D. Van Der Spoel, E. Lindahl, B. Hess, G. Groenhof, A. E. Mark, and H. J. Berendsen, "GROMACS: fast, flexible, and free," Journal of computational chemistry, vol. 26, no. 16, pp. 1701-1718, 2005.

[26] M. J. Abraham et al., "GROMACS: High performance molecular simulations through multi-level parallelism from laptops to supercomputers," SoftwareX, vol. 1, pp. 19-25, 2015.

[27] M. J. Abraham, D. van der Spoel, E. Lindahl, and B. Hess, GROMACS User Manual version 5.1-beta1, [Online]. Available: ftp://ftp.gromacs.org/pub/manual/manual-5.1-beta1.pdf

[28] W. Humphrey, A. Dalke, and K. Schulten, "VMD: visual molecular dynamics," Journal of molecular graphics, vol. 14, no. 1, pp. 33-38, 1996.

[29] C. Reichardt and T. Welton, Solvents and solvent effects in organic chemistry. John Wiley \& Sons, 2011. 